\title{
Artificially anisotropic cylinder to convert omnidirectional linear polarization into circular polarization
}

\author{
Emmi Kaivanto, Erkki Salonen and Markus Berg \\ Electrical Engineering Department, Centre for Wireless Communications, University of Oulu, FIN-90014, Finland (email: \\ emmi.kaivanto@oulu.fi)
}

Keywords: artificially anisotropic material, antenna polarisation, circular polarisation.

\begin{abstract}
Circular polarization and omnidirectional radiation are desired characteristics in various mobile communication systems. This paper studies possibilities to convert linear polarization into circular polarization by designing an artificially anisotropic cylinder around the antenna. The design process and simulated results for the cylinder with a half wave dipole antenna at the centre frequency of $2.3 \mathrm{GHz}$ are presented. It is shown that the cylinder has minor effect on the return loss and gain of the antenna and that axial ratio remains below $5 \mathrm{~dB}$ over the frequency range of $900 \mathrm{MHz}$.
\end{abstract}

\section{Introduction}

One of the most intensely researched area at the time being is the 5th generation network technology, which most probably will utilize frequency bands at millimeter frequencies. At those frequencies path losses increase significantly which inevitably leads to short range and line of sight communication. Hence, circular polarization is said to be indispensable for robust wireless communication between mobile devices at mm-waves [1]. To date circular polarized antennas have been widely used in communication systems for rescue workers, intelligent transportation systems, space and military communications, health monitoring systems, navigation, radar and satellite systems.

In the case of linearly polarized antennas the mutual orientation of the antennas matters. Polarization loss is present everywhere except when the antennas -or their field vectors - are parallel to each other. When the antennas are orientated perpendicularly, the signal may be lost completely. In the case of circular polarization the polarization loss does not depend on the orientation of the antennas, whereas the direction of rotation of the propagating wave is more important.

There are several ways to create a circularly polarized wave: By designing an antenna element which produces circular polarization such as a patch, a disc or a conical horn [2] - [4], by using a suitable feeding mechanism for linearly polarized antennas or an antenna array [5] - [7] or by using artificially anisotropic materials [8]. Nevertheless, the goal is to simultaneously excite two orthogonal electrical field vectors of equal amplitude with phase difference of $+/-90^{\circ}$.
This paper investigates the possibility to convert the radiation of an omnidirectionally radiating, linearly polarized antenna into circular polarization by designing an artificially anisotropic 3D printable cylinder around a dipole antenna. This study is made for a half wavelength dipole operating at 2.3 $\mathrm{GHz}$ but the structure can be designed at any frequency.

\section{Design of the artificially anisotropic cylinder}

\subsection{Theory}

When an electromagnetic wave is linearly polarized, its two orthogonal electric field components are in the same phase (or $180^{\circ}$ out of phase), whereas circular polarization can be achieved only when the magnitudes of the components are equal and the phase difference is $90^{\circ}$ [9]. Hence, to be able to turn linear polarization into circular polarization, one need to arrange the $90^{\circ}$ phase shift between the field components.

On the other hand it is known that the propagation speeds of the two orthogonal electric field components are different in an anisotropic material which dielectric constant differ in those two orthogonal directions. Figure 1 describes an anisotropic material which is made by stacking thin sheets of a dielectric material with air gaps. When a linearly polarized wave meets the sheets in the angle of $45^{\circ}$ the perpendicular electric field component travels slower through the material than the orthogonal component. Hence, the desired phase shift can be achieved for a certain thickness of the material.

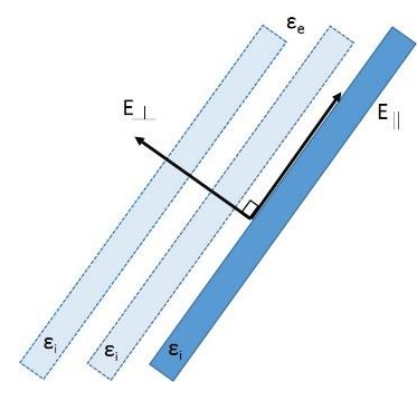

Figure 1. The orthogonal electric field components in the artificially anisotropic material in which the permittivity of environment is marked with $\varepsilon_{\mathrm{e}}$ and the permittivity of the inclusion material with $\varepsilon_{\mathrm{i}}$. 
The phases of the electric components on the both sides of the artificially anisotropic material are described in Figure 2.

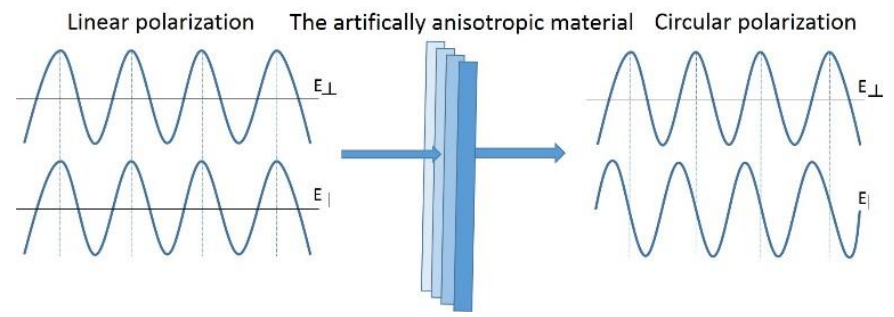

Figure 2. The effect of the artificial anisotropic material on the phases of the electric field components.

The starting point for the design was in Maxwell Garnett mixing formulas for ellipsoidal inclusions. According to [10] and [11], a disc can be considered as a "flatten ellipsoid" in which case the formulas for the parallel and perpendicular values for the effective permittivity of the thin sheets simplify as follows:

$$
\begin{aligned}
& \varepsilon_{\perp}=\frac{\varepsilon_{e} \varepsilon_{i}}{v_{f} \varepsilon_{e}+\left(1-v_{f}\right) \varepsilon_{i}} \\
& \varepsilon_{\|}=v_{f} \varepsilon_{i}+\left(1-v_{f}\right) \varepsilon_{e}
\end{aligned}
$$

The parameter $v_{\mathrm{f}}$ is the volume fraction which describes the share of the inclusion material $\varepsilon_{\mathrm{i}}$ of total volume.

To achieve exactly the desired $90^{\circ}$ phase shift between the orthogonal electric field components, the wave must travel in the material for a certain length. In this case the length is the thickness $D$ of the cylinder wall and it can be calculated from the equation (3):

$$
D=\frac{(P / 2 \pi) \cdot \lambda}{\sqrt{\varepsilon_{\|}}-\sqrt{\varepsilon_{\perp}}}
$$

where $\lambda=c / f$ and $c$ is the speed of light and $f$ is the desired central frequency [8]. In the case of circular polarization the lowest thickness can be achieved for $P=\pi / 2$ and hence the equation (3) simplifies to:

$$
D=\frac{\lambda}{4 \cdot\left(\sqrt{\varepsilon_{\|}}-\sqrt{\varepsilon_{\perp}}\right)}
$$

The cylindrical coordinate system $(\rho, \varphi, z)$ was used to describe geometry of the anisotropic material around the dipole antenna. The $45^{\circ}$ tilt angle of the sheets observed from the vertical axis of the antenna to the perpendicular direction can be achieved from the differential equation

$$
\mathrm{d} z=\rho \cdot \mathrm{d} \varphi
$$

By integrating the equation, the surfaces for the sheets can be defined as:

$$
z=\rho\left(\varphi+\varphi_{\mathrm{ij}}\right)
$$

where $z \in[-\mathrm{h} / 2, \mathrm{~h} / 2]$ and $\rho \in\left[\mathrm{R}_{\mathrm{in}}, \mathrm{R}_{\text {out }}\right]$ and $h$ is the height of the cylinder, $R_{\text {in }}$ and $R_{\text {out }}$ are the inner and outer radius of the cylinder and

$$
\begin{aligned}
& \varphi_{\mathrm{i} 0}=\mathrm{i} \cdot 2 \pi / \mathrm{N} \\
& \varphi_{\mathrm{i} 1}=\left(\mathrm{i}+\mathrm{v}_{\mathrm{f}}\right) \cdot 2 \pi / \mathrm{N},(\mathrm{i}=0 \ldots \mathrm{N}-1)
\end{aligned}
$$

where $\mathrm{N}$ is the number of sheets. The space between two surfaces defined by $\varphi_{\mathrm{i} 0}$ and $\varphi_{\mathrm{i} 1}$ is then filled with dielectric material.

To be able to research the function of the cylinder, a linearly polarized, omnidirectionally radiating half wave dipole was chosen to act as the radiator inside the cylinder. The center frequency of $2.3 \mathrm{GHz}$ was chosen because it was the frequency of the best available dipole antenna.

\subsection{Matching layer}

Reflections tend to cause losses and polarization distortion particularly for high permittivity materials. One method to reduce the total reflection is to use quarter wave matching layers, where the first penetrated field component and all multireflected component forwards are in the same phase. For the circular polarizer both parallel and perpendicular field components cannot be matched simultaneously. Hence, as the effective permittivity in parallel direction (2) is much higher than in perpendicular direction (1), the matching was dimensioned for the parallel component. The effective permittivity $\varepsilon_{M}$ for the matching layer can be calculated by the means of the equation for the quarter wave matching:

$$
\eta_{M}=\sqrt{\eta_{0} \eta_{\|}}=\eta_{0} \frac{1}{\sqrt[4]{\varepsilon_{\|}}}
$$

in which $\eta_{0}$ is the wave impedance in air and $\eta_{\|}$is the parallel wave impedance in the material. The equation yields $\varepsilon_{M}=$ $\sqrt[4]{\varepsilon_{\|}}$. The thickness of each matching layer was designed to be

$$
D_{M}=\lambda /\left(4 \cdot \sqrt[4]{\varepsilon_{\|}}\right)
$$

The volume fraction for the matching layers was then calculated from the formula (2). Because the matching layer is also anisotropic, the thickness of the center layer can be reduced by applying formulas (1)-(3). The more sparse inner layer also decreases the effect of the cylinder on the antenna matching.

The permittivity of the inclusion material was chosen to be $\varepsilon_{\mathrm{i}}=4$ as it is the permittivity of a material available for the $3 \mathrm{D}$ printer to be used. All chosen and calculated design parameters and dimensions are listed in Table 1.

\begin{tabular}{|l|l|l|l|l|l|l|l|}
\hline$\varepsilon_{\mathrm{e}}$ & $\varepsilon_{\mathrm{i}}$ & $\mathrm{V}_{\mathrm{f}, \mathrm{M}}$ & $\mathrm{V}_{\mathrm{f}, \mathrm{S}}$ & $\varepsilon_{\perp}$ & $\varepsilon_{\|}$ & $\mathrm{D}$ & $\mathrm{D}_{\mathrm{M}}$ \\
\hline 1 & 4 & 0.5 & 0.2 & 1.6 & 2.5 & $97 \mathrm{~mm}$ & $24 \mathrm{~mm}$ \\
\hline
\end{tabular}

Table 1. The design parameters and dimensions 


\subsection{Simulation results}

The function of the cylinder was researched by simulations. The simulation software used was CST Microwave Studio. The final structure with the matching layers is depicted in Figure 3. To minimize effects on the matching of the dipole, a small gap was left between the radiator and the cylinder. The radius of the inner circle is $7 \mathrm{~mm}$.

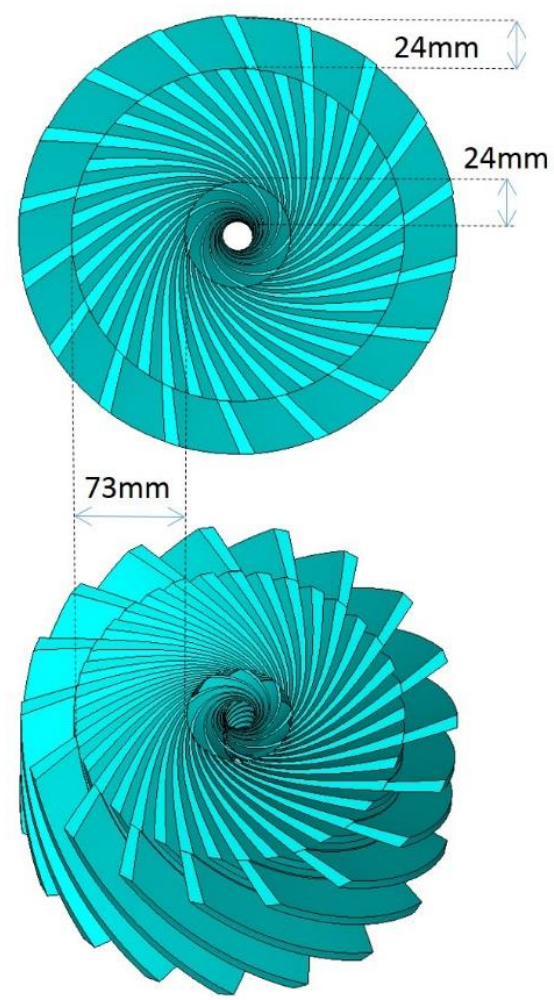

Figure 3 . The structure of the artificially anisotropic cylinder from above and from an upper angle.

Simulated S11 curves of the antenna without and with the cylinder are depicted in Figure 4. Adding the cylinder shifts the frequency less than $100 \mathrm{MHz}$, otherwise the matching remains almost the same.

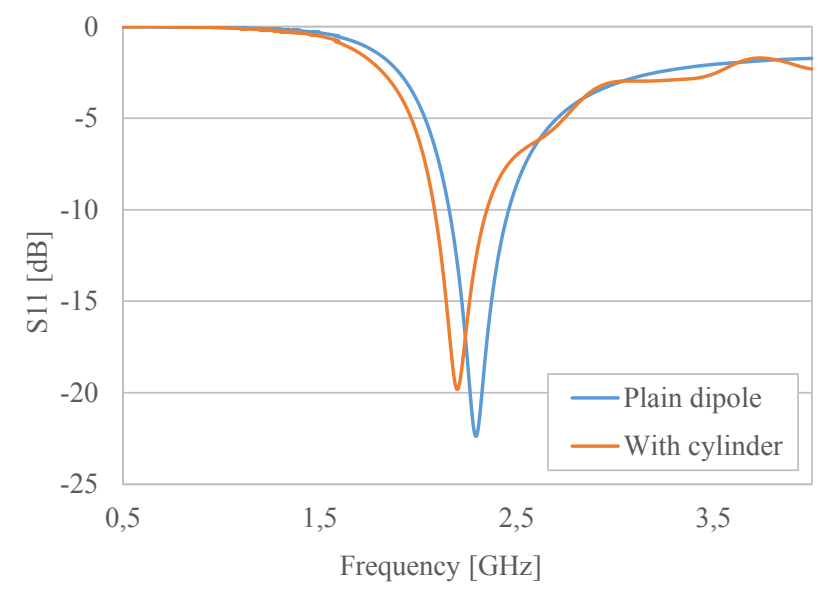

Figure 4. Simulated S11 curves for the dipole alone and the dipole with the artificially anisotropic cylinder.
The simulated 3D radiation patterns in Figure 5 without and with the cylinder at the center frequency of $2.3 \mathrm{GHz}$ show that the effect of the cylinder on the antenna performance is minor and the radiation pattern remains omnidirectional. The directivity is slightly increased and hence the maximum gain of the antenna is approximately $2 \mathrm{~dB}$ higher.

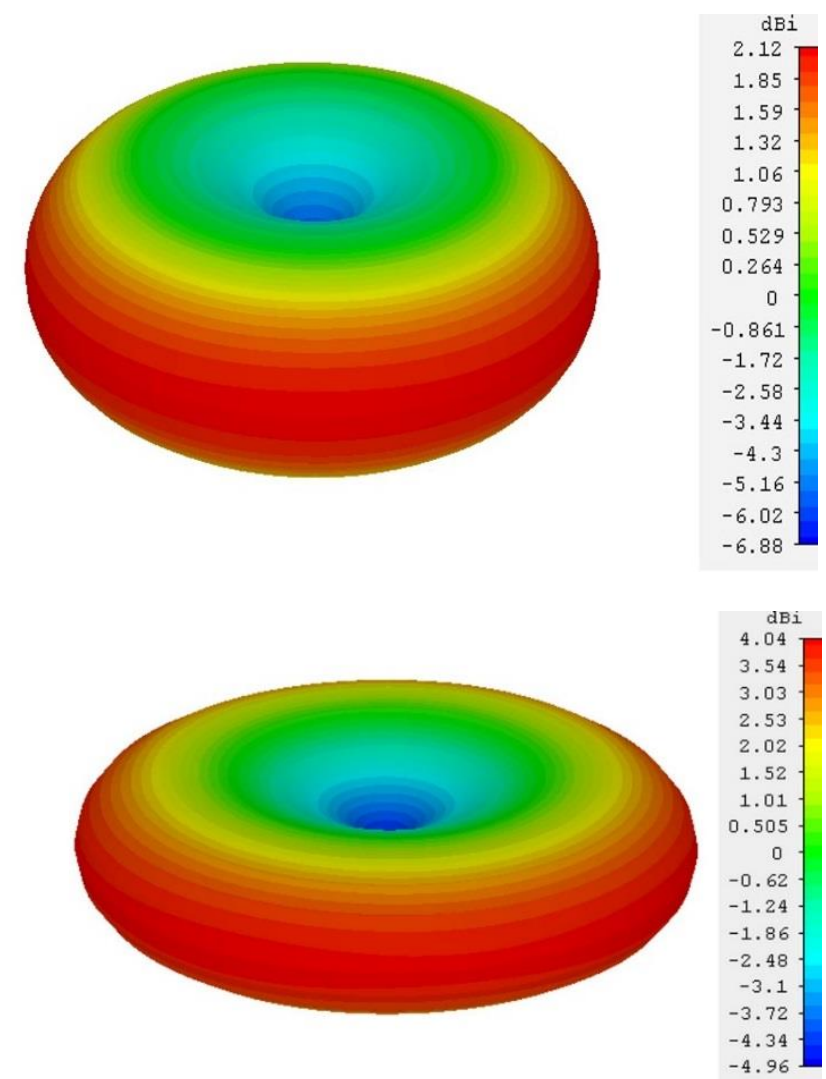

Figure 5. The simulated gain at $2.3 \mathrm{GHz}$ without and with the cylinder.

Simulated axial ratio at the center frequency of $2.3 \mathrm{GHz}$ is shown as a 3D plot in Figure 6. It can be seen that the polarization remains circular at wide angle. Figure 7 shows the axial ratio at three different frequencies.

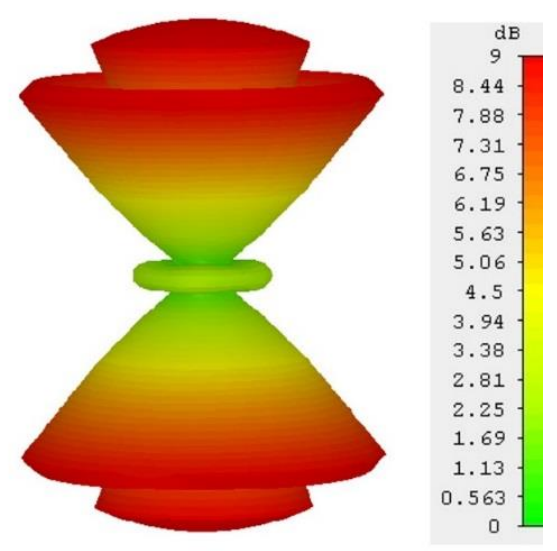

Figure 6 . The simulated $3 \mathrm{D}$ axial ratio at $2.3 \mathrm{GHz}$ 


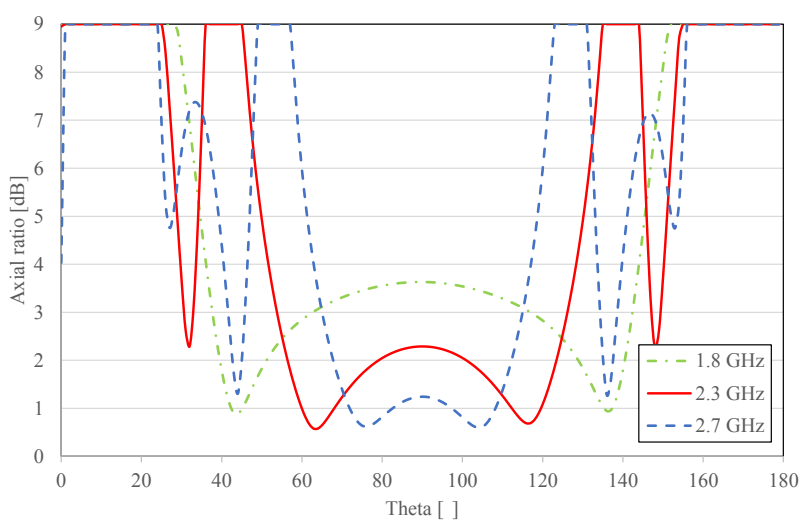

Figure 7. Simulated axial ratios at $1.8,2.3$ and $2.7 \mathrm{GHz}$ as a function of theta angle.

\section{Conclusion}

This paper studies possibilities to convert linear polarization into circular polarization by designing an artificially anisotropic cylinder around the antenna.

The structure includes quarter wave matching layers for reducing distortions caused by reflections. EM-simulations with a dipole antenna in the middle of the designed cylinder show that an omnidirectional circularly polarized radiation pattern with excellent axial ratio over wide band is achieved. The future work will include manufacturing a prototype using a 3D-printer and validation measurements.

\section{References}

[1] A. B. Smolders, U. Johannsen, "Axial Ratio Enhancement for Circularly-Polarized MillimeterWave Phased-Arrays Using a Sequential Rotation Technique", in IEEE Transactions on Antennas and Propagation, vol. 59, no. 9, pp. 3465-3469, (2011).

[2] D. Yu, S. X. Gong, Y. T. Wan, Y. L. Yao, Y. X. Xu, F. W. Wang, "Wideband Omnidirectional Circularly Polarized Patch Antenna Based on Vortex Slots and Shorting Vias", in IEEE Transactions on Antennas and Propagation, vol. 62, no. 8, pp. 3970-3977, (2014).

[3] P. Sharma, Gupta, "Analysis and optimized design of single feed circularly polarized microstrip antennas", in IEEE Transactions on Antennas and Propagation, vol. 31, no. 6, pp. 949-955, (1983).

[4] J. Huang, "Circularly polarized conical patterns from circular microstrip antennas," in IEEE Transactions on Antennas and Propagation, vol. 32, no. 9, pp. 991994, (1984).

[5] B. Y. Toh, R. Cahill, V. F. Fusco, "Understanding and measuring circular polarization", in IEEE Transactions on Education, vol. 46, no. 3, pp. 313318, (2003).
[6] J. Huang, "A technique for an array to generate circular polarization with linearly polarized elements", in IEEE Transactions on Antennas and Propagation, vol. 34, no. 9, pp. 1113-1124, (1986).

[7] M. A. Joyal, R. El Hani, M. Riel, Y. Demers and J. J. Laurin, "A Reflectarray-Based Dual-Surface Reflector Working in Circular Polarization", in IEEE Transactions on Antennas and Propagation, vol. 63, no. 4, pp. 1306-1313, (2015).

[8] T. Tuovinen, E. T. Salonen, M. Berg, "An Artificially Anisotropic Antenna Substrate for the Generation of Circular Polarization", in IEEE Transactions on Antennas and Propagation, vol. 64, no. 11, pp. 4937 4942, (2016).

[9] C. Balanis, “Antenna Theory, Analysis and Design", 3rd edition, New Jersey: Wiley, 2005.

[10]A. Sihvola, "Electromagnetic Mixing Formulas and Applications", IET Electromagnetic Waves Series, vol. 47, (2008).

[11] A. H. Sihvola, J. A. Kong, "Effective permittivity of dielectric mixtures", in IEEE Transactions on Geoscience and Remote Sensing, vol. 26, no. 4, pp. 420-429, (1988). 